\title{
Caltech-NRAO Stripe 82 Survey (CNSS). IV. The Birth of Radio-loud Quasar $013815+00$
}

\author{
Magdalena Kunert-Bajraszewska ${ }^{1}$ (D), Aleksandra Wołowska ${ }^{1}$, Kunal Mooley ${ }^{2,3}$ (D), Preeti Kharb ${ }^{4}$ (D), and Gregg Hallinan ${ }^{3}$ \\ ${ }^{1}$ Institute of Astronomy, Faculty of Physics, Astronomy and Informatics, NCU, Grudziadzka 5/7, 87-100, Torun, Poland; magda@astro.uni.torun.pl \\ ${ }_{2}$ National Radio Astronomy Observatory, Socorro, NM 87801, USA \\ ${ }^{3}$ Cahill Center for Astronomy, MC 249-17, California Institute of Technology, Pasadena, CA 91125, USA \\ ${ }^{4}$ National Centre for Radio Astrophysics (NCRA)-Tata Institute of Fundamental Research (TIFR), S. P. Pune University Campus, Post Bag 3, Ganeshkhind, Pune \\ 411007, India \\ Received 2020 April 5; revised 2020 May 14; accepted 2020 May 20; published 2020 July 9
}

\begin{abstract}
It is believed that the gas accretion onto supermassive black holes is the main process of powering this quasar's luminous emission, which occurs in optical, UV, and X-ray regimes and less frequently in radio waves. The observational fact that only a few percent of quasars are radio-loud is still an unresolved issue concerning the understanding of the active galactic nucleus (AGN) population. Here we present a detection of a rapid transition from the radio-quiet to the radio-loud mode in quasar $013815+00(z=0.94)$ which coincides with changes of its UV-optical continuum and the low ionization Mg II broadline. We interpret this as an enhancement of accretion onto a central black hole of about $10^{9}$ solar masses. As a consequence a new radio-loud AGN was born. Its spectral and morphological properties indicate that it went through the short gigahertz-peaked spectrum phase at the beginning of its activity and has now stabilized its flux density at the level of a few millijansky. The radio morphology of $013815+00$ is very compact and we predict that with such short-term jet activity its development will be very slow. The observed luminosity changes of the accretion disk are shorter than the lifetime of the new radio phase in $013815+00$.
\end{abstract}

Unified Astronomy Thesaurus concepts: Active galactic nuclei (16); Radio active galactic nuclei (2134)

\section{Introduction}

The radio emission of active galactic nuclei (AGNs) is dominated mainly by synchrotron radiation of electrons accelerated by powerful jets. However, such AGNs traditionally called radio-loud constitute only a small part of the entire population. About $90 \%$ of AGNs are much fainter in the radio and they are called radio-quiet sources (Kellerman et al. 1989). The origin of their radio emission is still unclear and widely debated as well as the nature of the event that sparks the radio-loud phase. In general, source classification for radio-loud/radio-quiet is a matter of the definition used and since it is based on selected observational properties it can be somewhat ambiguous (Kharb et al. 2014; Foschini 2017). The presence or absence of a strong relativistic jet seems to be a better criterion for differentiating these two groups of objects (Padovani 2017). However, it has its limitations since it cannot be used in the case of unresolved sources with faint radio flux densities.

According to the generally accepted model (Fanti et al. 1995; Readhead et al. 1996; O’Dea \& Baum 1997) the radio sources having a peak in their radio spectra above $1 \mathrm{GHz}$ represent the early stages of the life cycle of radio-loud sources. These are the compact $(<1 \mathrm{kpc})$ gigahertz-peaked spectrum (GPS) and highfrequency-peaked sources. The latter group are sources with peaks above $5 \mathrm{GHz}$ (Dallacasa et al. 2000), but the peak value may vary, and that is why in the literature these objects are often referred to as GPSs. The peak emission frequency is inversely proportional to the source size (O'Dea \& Baum 1997) showing that the next in this development sequence are compact steep spectrum (CSS) sources. CSSs have larger linear sizes $(\sim 1-20 \mathrm{kpc})$ and peak frequencies below $1 \mathrm{GHz}$. The ability to perform sensitive observations at low radio frequencies has recently revealed many objects with spectral peaks observed at frequencies far below $1 \mathrm{GHz}$ called megahertz-peaked spectrum sources (Callingham et al. 2017; Keim et al. 2019). The final stage of the life cycle is a large scale $(>20 \mathrm{kpc})$ radio-loud AGN of Fanaroff-Riley I (FRI) or II (FRII) morphology (Fanaroff and Riley 1974).

Recent reports show that the GPS and CSS sources account for only $2 \%$ of the brightest AGNs (Sotnikova et al. 2019). But in the case of low luminosity objects the compact population is much larger and even makes up the majority of samples tested (Sadler et al. 2014; Baldi et al. 2015). This excess of small AGNs in relation to "adult" objects gave rise to the suggestion that not all compact young sources evolve into extended objects. According to one of the hypotheses the radio activity is a short-term phenomenon for many AGNs lasting $10^{4}-10^{5} \mathrm{yr}$, and an AGN may undergo numerous such short phases during its lifetime (Reynolds \& Begelman 1997; Czerny et al. 2009; KunertBajraszewska et al. 2010; An \& Baan 2012; Wołowska et al. 2017). The reason for this premature termination of activity could be, e.g., instabilities of the accretion disk that cause the jet disruption (Czerny et al. 2009). However, there are also results indicating that at least for some low luminosity AGNs their compact radio morphology does not mean a young age but rather suggests a presence of different fueling mechanisms (Capetti et al. 2019; Hardcastle et al. 2019). Regardless of the hypotheses presented above, the GPS phase seems to be the starting point for the development of each new phase of radio activity and is associated with the emergence of a new radio jet.

The first detection of switched-on radio activity was in a $z=1.65$ quasar, VTC 233002-002736, found in the pilot Caltech-NRAO Stripe 82 Survey (CNSS) carried out over a 50 $\mathrm{deg}^{2}$ region of Stripe 82 (CNSS Paper I; Mooley et al. 2016). We reported a GPS-like characteristic of its radio spectrum, typical for a young source. The burst of radio activity has been also discovered recently in radio-quiet narrow-line Seyfert 1 


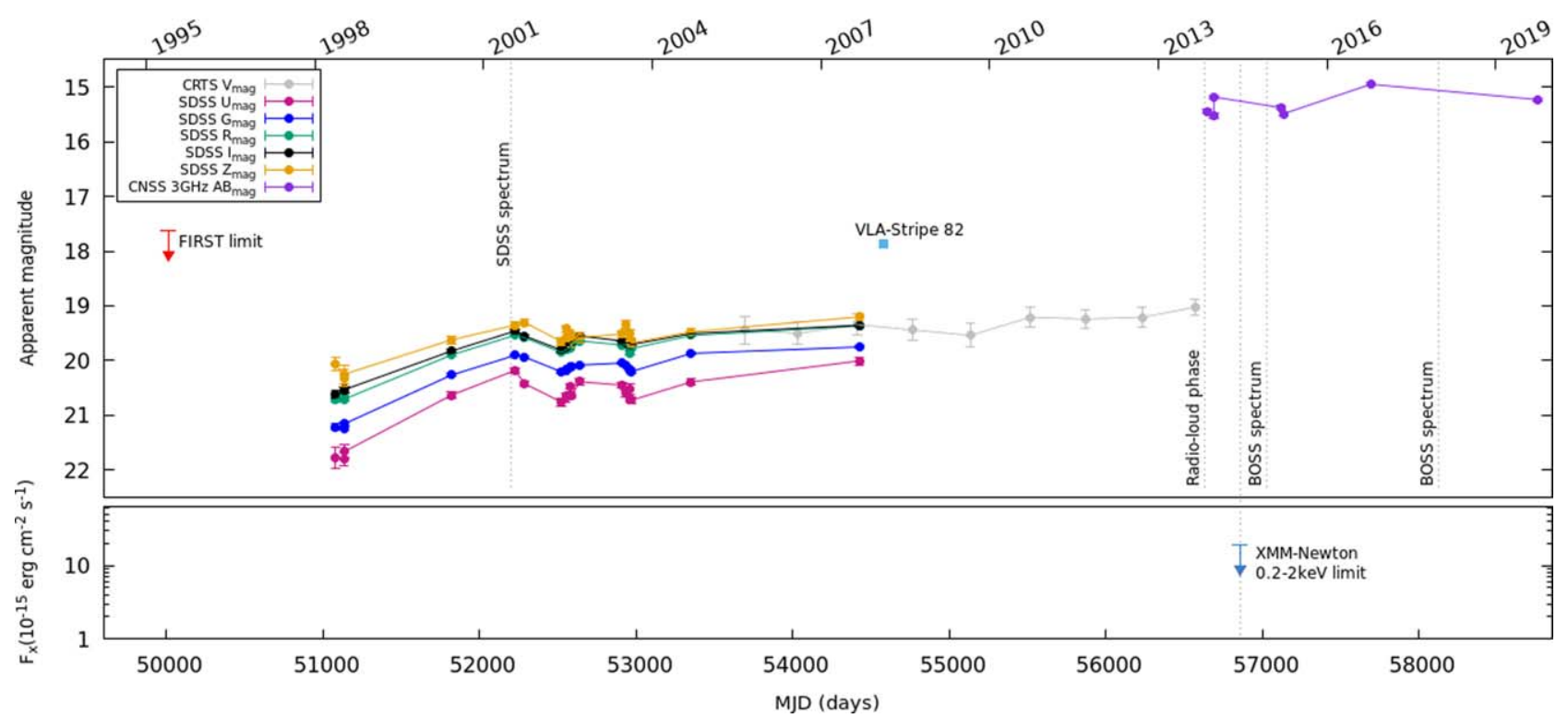

Figure 1. Top panel: long-term optical light curve for SDSS J0138+0029. The SDSS photometric measurements in the $u^{\prime}, g^{\prime}, r^{\prime}, i^{\prime}, z^{\prime}$ filters are shown with small filled circles. The three epochs of SDSS spectroscopy as well as the date of the radio activity burst are indicated by the dotted lines. The yearly median and rms of the unfiltered CRTS measurements are shown with gray filled circles. The radio measurements have been converted to apparent magnitude on the AB system of Oke \& Gunn (1983) and they are the FIRST $3 \sigma$ rms noise level (red arrow), the VLA-Stripe 82 source emission (blue square), the 3 GHz light curve detected by CNSS, and later 2016 and 2019 VLA measurements (purple circles). Bottom panel: the upper limit of the X-ray flux from XMM-Newton observations, which amounts to $F_{0.2-2 \mathrm{keV}}=18.0 \times 10^{-15} \mathrm{erg} \mathrm{cm}^{-2} \mathrm{~s}^{-1}$ (LaMassa et al. 2013).

(NLS1) galaxies (Lähteenmäki et al. 2018). This is interpreted as the launch of a relativistic jet indicating the similarity in the radio behavior of NLS1 galaxies and more energetic AGNs.

In this paper we present the first few years of development of the radio-loud phase of another quasar, CNSS J013815+002914 (hereafter $013815+00$ ). The radio emission of $013815+00$ was first discovered in the CNSS survey, on 2013 December 20 at $3 \mathrm{GHz}$. We report multiepoch and multifrequency observations of this source in Section 2. We found that the rapid changes of radio spectrum of quasar $013815+00$ transforming the initial GPS shape into a flat spectrum are associated with the changes of brightness in the AGN accretion disk. The high-resolution Very Long Baseline Array (VLBA) observations confirm that the burst of radio activity is associated with the appearance of a small radio jet. We discuss these findings and implications in detail in Section 3 and summarize our work in Section 4.

Throughout this paper we assume a cosmology in which $H_{0}=70 \mathrm{~km} \mathrm{~s}^{-1} \mathrm{Mpc}^{-1}, \Omega_{m}=0.3$ and $\Omega_{\lambda}=0.27$. The radio spectral index $\alpha$ is defined in the sense $\mathrm{S} \propto \nu^{\alpha}$.

\section{Observations and Data Analysis}

\subsection{Radio Data}

The radio emission of quasar $013815+00$ (R.A. $01^{\mathrm{h}} 38^{\mathrm{m}} 15^{\mathrm{s}} .061$, decl. $00^{\mathrm{d}} 29^{\mathrm{m}} 14^{\mathrm{s}} .07$ ) was detected on 2013 December 20 at $3 \mathrm{GHz}$ by the CNSS, a multiepoch radio transient survey carried out between 2012 December and 2015 May with the Jansky Very Large Array (VLA; Mooley et al. 2016). It was designed for systematically exploring the radio sky for slow transient phenomena on timescales between one day and several years.

All the calibration process and imaging of these observations was done using a custom-developed, semiautomated AIPSLite/Python-based pipeline developed at Caltech and CASA ${ }^{5}$. The observed $3 \mathrm{GHz}$ flux density of quasar $013815+00$ was

\footnotetext{
5 http://casa.nrao.edu
}

$2.41 \pm 0.09, \quad 2.25 \pm 0.09, \quad 3.07 \pm 0.09, \quad 2.56 \pm 0.09, \quad$ and $2.31 \pm 0.09 \mathrm{mJy}$ in the five epochs of observation indicating relatively stable behavior (Figure 1).

After the discovery of radio quasar $013815+00$ we performed its follow-up wide range observations with the JVLA in A configuration on 2016 November 16 (project VLA/16B-047, $6 \mathrm{hr}$ ), using five receivers covering the spectrum from 1000 to $16884 \mathrm{MHz}(\mathrm{L}, \mathrm{S}, \mathrm{C}, \mathrm{X}$, and $\mathrm{Ku}$ ). The observing setup was the correlator with 16 spectral windows and $642 \mathrm{MHz}$-wide channels. Another epoch of observations was obtained in 2019 November (project VLA/19B-209, $6 \mathrm{hr}$ ) in D configuration using four receivers $(S, C, X$, and $\mathrm{Ku})$ and the same observing setup. Then the detailed calibration and imaging of VLA data was carried out using CASA.

The high-resolution observations of $013815+00$ were made with the VLBA at $C$-band on 2016 February 26. The data were correlated at the Array Operations Center in Socorro (USA). We phase-referenced our observations to the VLBA calibrator $0137+012$. We used the strong source $\mathrm{J} 0237+2848$ as fringe finder and bandpass calibrator. In order to carry out the spectral analysis of the radio components of our source we divided the available bandwidth at $C$-band receiver into two subbands centered at 4.5 and $7.5 \mathrm{GHz}$. This strategy allowed us to obtain images at two frequencies in one scan. Data reduction (including editing, amplitude calibration, instrumental phase corrections and fringefitting) was performed with the standard procedure using the NRAO AIPS ${ }^{6}$ software. After this stage the AIPS task IMAGR was used to produce the final total intensity images. The flux densities of the main components of the target source were then measured by fitting Gaussian models using AIPS task JMFIT. The linear size of the source was calculated based on the largest angular size measured in the $4.5 \mathrm{GHz}$ image contour plot.

In order to complement the spectrum of $013815+00$ at low frequencies, sub-GHz observations with upgraded GMRT in

\footnotetext{
6 http://www.aips.nrao.edu
} 
$013815+00$

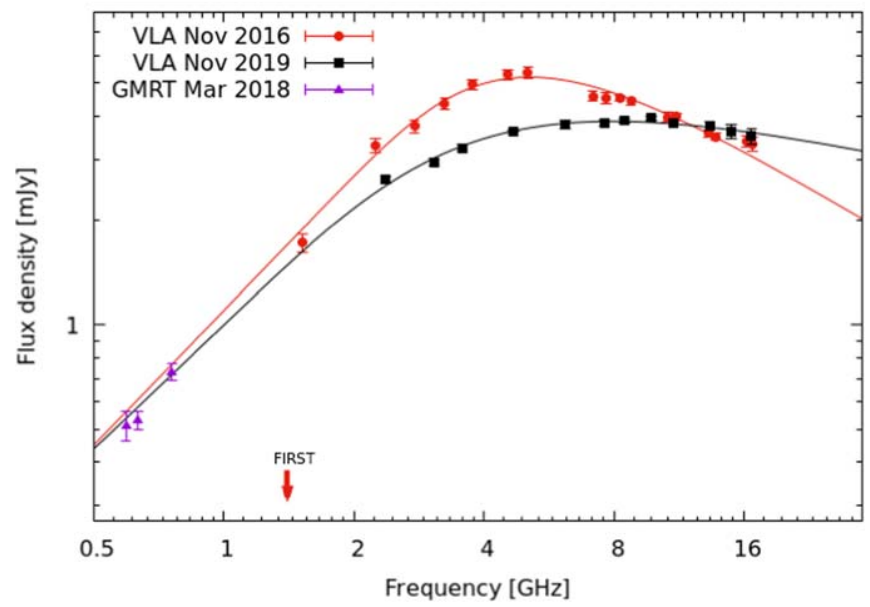

Figure 2. Radio spectrum of quasar $013815+00$ consisting of VLA measurements in the range $1-20 \mathrm{GHz}$ and low frequency GMRT observations in the range $0.6-0.8 \mathrm{GHz}$. The red arrow indicates the $3 \sigma$ upper limit at $1.4 \mathrm{GHz}$ from the FIRST survey.

Band 3 and Band 4 were carried out on 2018 March 16 and 19. Unfortunately, Band 3 data were corrupted, probably due to incorrect phase calibration. However, good quality Band 4 (560-810 MHz) data were obtained, and analyzed with CASA software. In order to obtain as many measurement points as possible, while maintaining a sufficient signal-to-noise $(\mathrm{S} / \mathrm{N})$ ratio, the data were divided into two spectral windows, each one processed separately and added to the radio spectrum plot (Figure 2).

Finally, in order to characterize the significant changes in the radio spectrum of $013815+00$ it has been fitted with a modified power-law model (Snellen et al. 1998):

$$
S(\nu)=\frac{S_{p}}{\left(1-e^{-1}\right)} \times\left(\frac{\nu}{\nu_{\mathrm{p}}}\right)^{\alpha_{\text {thick }}} \times\left(1-e^{-\left(\frac{\nu}{\nu_{\mathrm{p}}}\right)^{\alpha_{\mathrm{thin}}-\alpha_{\mathrm{thick}}}}\right),
$$

where $\alpha_{\text {thick }}$ is the optically thick spectral index, $\alpha_{\text {thin }}$ is the optically thin spectral index, and $S_{p}$ and $\nu_{p}$ are the peak flux density and peak frequency, respectively. Since the GMRT observations were carried out in a different time than the VLA observations, only VLA data were fitted in each epoch. However, the low frequency GMRT data are also indicated on the plot. The fits characterize the significant changes that occur in the optically thin part of spectrum of quasar $013815+00$. At lower frequencies, as might be expected, the spectrum is more stable. It is also very likely that there are no changes at GMRT frequencies. Therefore, the location of these points in relation to the modeled VLA radio spectrum curve influenced the choice of the best model. In addition, to obtain a satisfactory fit to the VLA data we had to limit the number of free parameters by one of the spectral indices.

\subsection{Optical Observations}

To track the brightness changes in the optical and UV range of quasar $013815+00$ we have collected all available photometric data points since 1998 until the end of 2013 from the Sloan Digital Sky Survey (SDSS) and Catalina Sky Survey (CRTS; Drake et al. (2009). We averaged the CRTS data in segments of $1 \mathrm{yr}$ and put the yearly median and rms values on Figure 1 together with the SDSS data.

Additionally, three epochs of SDSS spectroscopic measurements of $013815+00$ are available and were analyzed in this article. The first spectroscopic observation was made by the SDSS on 2001 October 21 over the wavelength range of $3800-9200 \AA$ at a spectral resolution of $\simeq 2000$. The other two were made by the Extended Baryon Oscillation Spectroscopic Survey (eBOSS) over the slightly different than previous wavelength range $3600-10,000 \AA$ at a spectral resolution of $\simeq 2000$ on 2015 December 8 and 2018 January 6 .

The SDSS spectra were corrected for Galactic extinction with the reddening map of Schlafly \& Finkbeiner (2011), and shifted to the rest-frame wavelength by using the SDSS redshift. The decomposition of the spectra has been done using the IRAF package and assuming the following components: power law (representing the emission from an accretion disk), Fe II pseudocontinuum, and suite of Lorentzian and Gaussian components to model the broad and narrow emission lines. In order to remove the contribution from Fe II emission, the iron template from Bruhweiler \& Verner (2008) was fitted to both spectra. The template was convolved with a Gaussian profile with different dispersion values for kinematic broadening of Fe II lines, in order to find the most accurate one. The emission lines were fitted with a Lorentzian/Gaussian function (with single or multiple components, depending on a line) to determine fluxes and FWHMs. The FWHM of the [O III] line quoted in Table 1 was obtained by fitting a single Gaussian to enable comparison of this value with those obtained for other compact radio objects. The quality of the spectra did not allow for more complex modeling of the [O III] $\lambda \lambda 4959,5007$ doublet.

The error estimation of the continuum flux density was made using the rms method. The uncertainty of the line flux measurements has been estimated using the standard formula for noise averaging $\sigma_{f}=\sigma_{c} L / \sqrt{N}$, where $\sigma_{c}$ is the $\mathrm{rms}$ of continuum flux density at $3000 \AA, L$ is the integration interval, and $N$ is the number of spectrum samples. The error of the line width has been calculated by finding minimum and maximum widths of the Lorentz/Gaussian line fit at which the integral of the fit changes by $\pm \sigma_{f}$ keeping the amplitude of the fit fixed. The properties of the broad and narrow lines resulting from the fit to each spectrum are listed in Table 1.

The black hole mass was estimated from the $\operatorname{Mg}$ II $\lambda 2800$ line, using the following relation (Trakhtenbrot \& Netzer 2012):

$$
\frac{M_{\mathrm{BH}}}{M_{\odot}}=5.6 \times 10^{6}\left(\frac{\lambda L_{3000}}{10^{44} \mathrm{erg} \mathrm{s}^{-1}}\right)^{0.62}\left[\frac{\mathrm{FWHM}(\mathrm{Mg} \mathrm{II})}{10^{3} \mathrm{~km} \mathrm{~s}^{-1}}\right]^{2} .
$$

\section{Discussion}

The radio emission of quasar $013815+00$, which is located in the Stripe82 region, was detected on 2013 December 20 at the level of few millijansky with VLA at $3 \mathrm{GHz}$. Previous surveys of this part of Stripe82 were carried out in 1995 and 2008 with VLA at $1.4 \mathrm{GHz}$ under the names the Faint Images of the Radio Sky at Twenty-Centimeters (FIRST; White et al. 1997) and the VLA survey of the SDSS Southern Equatorial Stripe (VLA-Stripe 82; Hodge et al. 2011), respectively. Both surveys did not report a detection of this quasar at the catalog detection limit at the source position of $0.78 \mathrm{mJy}$ (FIRST) and $0.67 \mathrm{mJy}$ (VLA-Stripe 82). However, a careful inspection of the radio images, revealed an unresolved weak emission at the location of $013815+00$ amounting to $0.26 \pm 0.06 \mathrm{mJy}$ in the case of more sensitive 
Table 1

Line and Continuum Measurements from SDSS/BOSS Spectra

\begin{tabular}{|c|c|c|c|}
\hline QSO $013815+00$ & 2001 & 2015 & 2018 \\
\hline $\mathrm{Mg}$ II flux $\left(10^{-17} \mathrm{erg} \mathrm{s}^{-1} \mathrm{~cm}^{-2}\right)$ & $145 \pm 15$ & $356 \pm 6$ & $307 \pm 8$ \\
\hline $\mathrm{Mg}$ II FWHM $\left(\mathrm{km} \mathrm{s}^{-1}\right)$ & $9428 \pm 1283$ & $9964 \pm 257$ & $9857 \pm 386$ \\
\hline $\mathrm{Mg} \mathrm{II} \log _{10}\left(L_{\mathrm{Mg} \mathrm{II}} / \mathrm{erg} \mathrm{s}^{-1}\right)$ & $42.81 \pm 0.05$ & $43.20 \pm 0.01$ & $43.14 \pm 0.01$ \\
\hline$M_{\mathrm{BH}}\left(10^{9} M_{\odot}\right)$ & $1.6 \pm 0.7$ & $2.3 \pm 0.2$ & $1.8 \pm 0.3$ \\
\hline$L_{\mathrm{bol}}\left(10^{44} \mathrm{erg} \mathrm{s}^{-1}\right)$ & $35.8 \pm 6.4$ & $50.6 \pm 2.9$ & $36.7 \pm 3.6$ \\
\hline [O III] $\lambda 5007$ flux $\left(10^{-17} \mathrm{erg} \mathrm{s}^{-1} \mathrm{~cm}^{-2}\right)$ & $\ldots$ & $42 \pm 2$ & $30 \pm 2$ \\
\hline
\end{tabular}

VLA-Stripe 82 observations. The radio image rms noise level at the position of our quasar amounts to $0.106 \mathrm{mJy}$ (FIRST) and $0.084 \mathrm{mJy}$ (VLA-Stripe 82). This indicates that in 2008 the emission of the $013815+00$ quasar was at the $3 \sigma$ rms noise level $(0.25 \mathrm{mJy})$ of the VLA-Stripe 82 survey. After the burst of radio activity, the latest measurement of its $1.4 \mathrm{GHz}$ flux density amounts to $1.61 \pm 0.11 \mathrm{mJy}$ and means about a six-fold increase in flux density at radio waves and a significant change in the value of radio-loudness parameter. To calculate the last we adopted a radio-loudness definition from Kimball et al. (2011): $\log R=$ $\left(M_{\text {radio }}-M_{i}\right) /-2.5$, where $M_{\text {radio }}$ is a radio absolute magnitude at $1.4 \mathrm{GHz}$ and $M_{i}$ is a Galactic reddening corrected $i$-band absolute magnitude. For the detection level of $0.26 \mathrm{mJy}$ (from VLA-Stripe 82) the value of the radio-loudness parameter amounts to $\log R=0.6$ and increases after the radio activity ignition to $\log R=1.4$ crossing the boundary $\log R=1$ above which the source is considered to be radio-loud.

\subsection{Analysis of Radio Properties}

The follow-up observations of quasar $013815+00$ performed with the VLA (1-15 GHz) on 2016 November 16 revealed a radio spectrum peaking at $\nu_{p}=4.72 \pm 0.19 \mathrm{GHz}$ with a flux density $\mathrm{S}_{p}=5.17 \pm 0.07 \mathrm{mJy}$ and the spectral index $\alpha_{\text {thin }}=-0.75$ and $\alpha_{\text {thick }}=1.3 \pm 0.1$ of the optically thin and thick parts of the spectrum, respectively (Figure 2). The $5 \mathrm{GHz}$ luminosity of this object at this epoch of observations has a moderate value of $\log _{10}\left[L_{5 \mathrm{GHz}} / \mathrm{W} \mathrm{Hz}^{-1}\right]=25.4$.

The VLBA high-resolution observations at 4.5 and $7.5 \mathrm{GHz}$ of this source performed on 2016 February 26 show a slightly resolved radio structure of the total projected linear size $l \approx 67 \mathrm{pc}$. The radio morphology indicates the presence of two compact features: (A) the central one with flux densities $2.14 \pm 0.07$ and $2.02 \pm 0.03 \mathrm{mJy}$, and (B) the northeastern component with flux densities $3.02 \pm 0.09$ and $2.80 \pm 0.03 \mathrm{mJy}$ at 4.5 and $7.5 \mathrm{GHz}$, respectively (Figure 3). The radio spectral index of both components between 4.5 and $7.5 \mathrm{GHz}$ is flat and amounts to -0.11 and -0.15 for the central and northeastern component, respectively. We suggest that the radio structure of $013815+00$ is a core-jet type with the central component being the radio core.

Over the next three years the radio morphology of quasar 013815+00 must undergo a rapid change, which is reflected in the significant evolution of its spectrum. The new VLA spectrum obtained on 2019 November 10 shows a drop in luminosity in the wide frequency range and spectral flattening of the thin part of the spectrum. The spectrum peaks at $\nu_{p}=4.21 \pm 0.20 \mathrm{GHz}$ with a flux density $\mathrm{S}_{p}=3.51 \pm 0.07 \mathrm{mJy}$ and the spectral index $\alpha_{\text {thin }}=$ $-0.27 \pm 0.03$ and $\alpha_{\text {thick }}=1.19$ of the optically thin and thick parts of the spectrum, respectively (Figure 2). We interpret the 2016 GPS spectrum of quasar $013815+00$ as a burst of new radio jet activity. As the radio jet expands, its flux density decreases and the spectrum starts to be dominated by the compact radio core. Further changes in the spectrum are still possible and only longterm monitoring will allow us to finally determine the shape of the spectrum. Current observations show that the source after a radioquiet phase will stabilize its radio flux density at the level of a few millijansky.

The radio properties of quasar $013815+00$ in its first phase of activity, namely the convex spectrum and compact morphology, make it follow the anticorrelation found by O'Dea \& Baum (1997) which relates the spectral turnover frequency (the frequency at which the spectrum peaks) in GPS and CSS sources to their projected linear sizes (Figure 4). There is a continuous distribution of these objects across the $\nu_{p}-1$ plane, which suggest that GPS and CSS sources are scaled versions of each other. However, the long-term monitoring of samples of GPS sources lasting 10-20 yr shows that with time even the majority of them cease to adhere to the generic GPS source properties (Dallacasa et al. 2000; Torniainen et al. 2005; Orienti et al. 2006; Sotnikova et al. 2019). This may be due to the fact that the GPS phase occurs in many, or even all, populations of radio sources and is what at the initial stage of development connects them with each other. In fact, however, these groups may not be related. In particular, this may apply to the division into galaxies and quasars. According to Snellen et al. (1999) GPS quasars can be a subclass of flat-spectrum quasars. Another complication in this model of the life cycle of radio source are the results from low luminosity AGNs, which show that these sources have different lifetime distribution (Capetti et al. 2019; Hardcastle et al. 2019).

013815+00 shares several properties similar to GPS quasars like convex spectrum and compact core-jet morphology, but has lower luminosity and its spectrum evolves rapidly. We thus conclude that $013815+00$ is a newborn radio source with a very short transient GPS phase. We suggest that the duration of the GPS phase may scale with the source luminosity and thus with the power of the jet.

Using the relation between the jet power and radio luminosity at $1.4 \mathrm{GHz}$ discussed by Rusinek et al. (2017) we estimated the power of the newborn jet in $013815+00$ to be $\log _{10}\left[P_{j} / \mathrm{erg} \mathrm{s}^{-1}\right]=44.1$. This value is intermediate, and it falls within a wide range of jet power values for young AGNs (Wójtowicz et al. 2020). In turn, such a wide distribution of jet power values may mean the existence of different subclasses of sources within the group of young radio sources (Berton et al. 2017; Fan \& Wu 2019), as we have already mentioned above. 

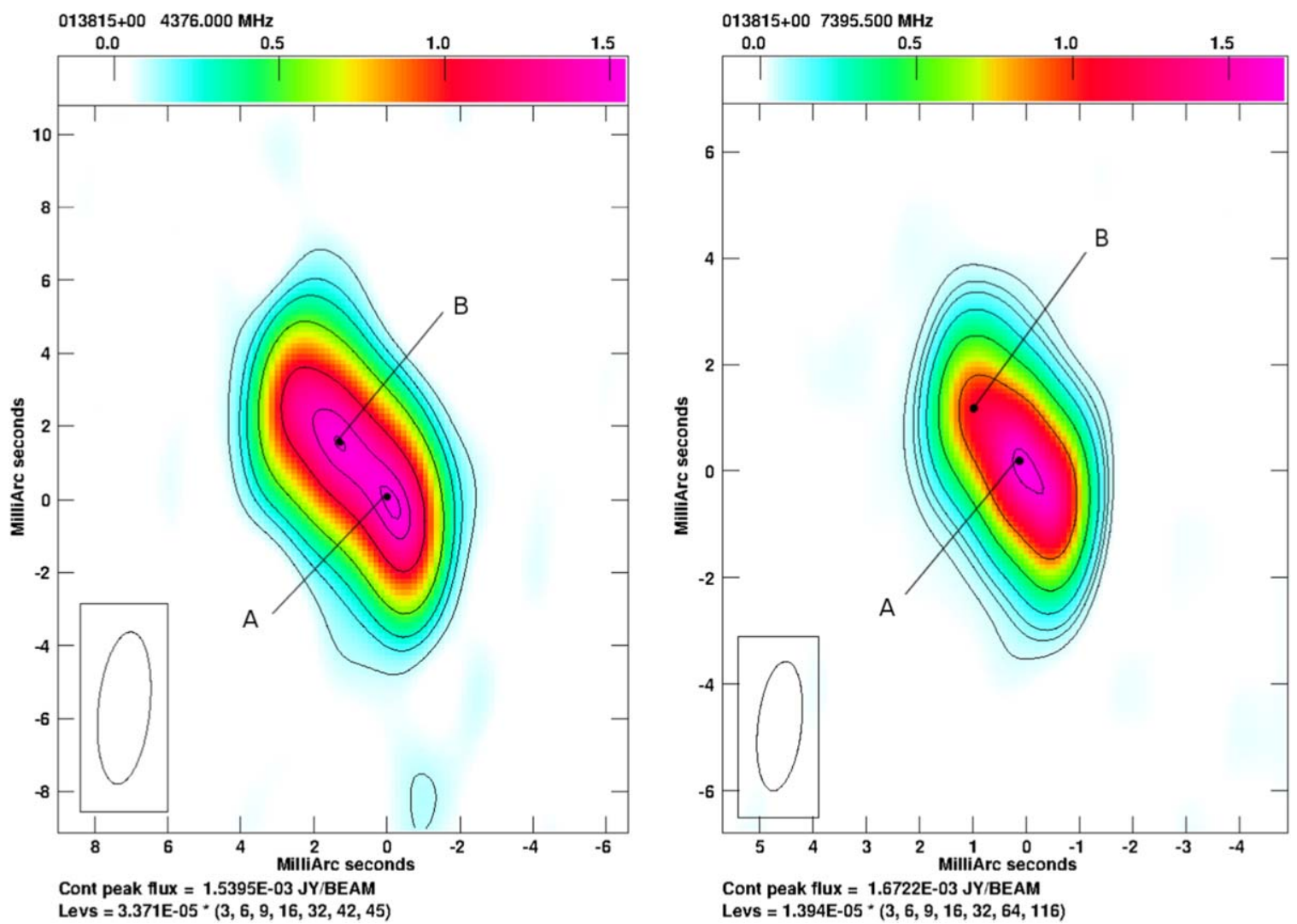

Figure 3. VLBA 4.5 and $7.5 \mathrm{GHz}$ images of quasar $013815+00$. The first contour level corresponds to $\approx 3 \sigma$ and the radio beam size is indicated in the lower left corner. The color bar shows the scale of intensity of radio emission in millijansky. The two identified radio components are indicated with dots.

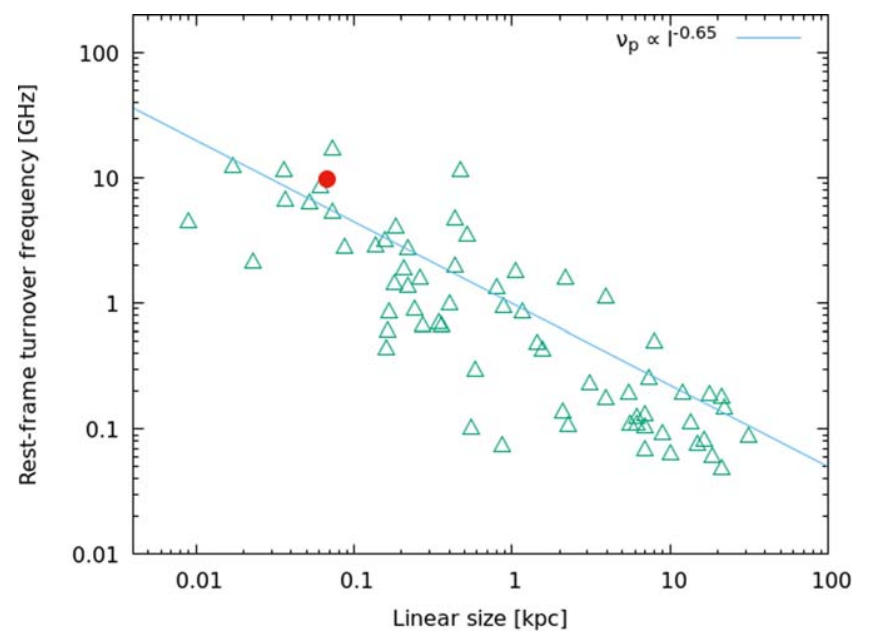

Figure 4. Rest-frame turnover frequency vs. projected linear size relation for CSS and GPS sources studied by O'Dea \& Baum (1997). The GPS quasar $013815+00$ is indicated with a red dot.

\subsection{Photometric and Spectroscopic Changes}

The collected photometric observations of quasar $013815+00$ from the period of $15 \mathrm{yr}$ show an over 1 magnitude brightening in all SDSS bands during the first three years of observations (Figure 1). At the peak of this brightening the first spectroscopic measurement of the source has been made by the SDSS on 2001 October 21. Over the next $12 \mathrm{yr}$ the quasar light curve shows a typical AGN submagnitude variability and slow but systematic increase in brightness of another $\sim 0.5$ magnitude in $V$-band of CRTS observations.
The other two spectroscopic observations were made by the eBOSS on 2015 December 8 and 2018 January 6. They took place after the burst of $013815+00$ radio activity in 2013 and enabled us to follow the changes in the continuum and the brightness of the emission lines that happened after this event. An increase of the level of continuum emission is clearly visible when comparing 2001 and 2015 spectra and amounts to 1.4 (Figure 5). We are not able to give a precise date of the beginning of this growth. However, the increased emission of the source in the optical-UV range continues for the next two years after the new radio jet launching in 2013. In period 2015-2018 the luminosity drops to the level close to that of the 2001 observations. During this time we also observe a significant change in the radio spectrum of quasar $013815+00$, which we interpret as a dissipation of jet energy as a result of its expansion.

\subsubsection{Mg II $\lambda 2800$ Line Behavior}

The value of redshift of $013815+00$ means that the Balmer emission lines are not in the SDSS (2001) spectrum but the $\operatorname{Mg}$ II $\lambda 2800$ line is clearly present. The BOSS $(2015,2018)$ spectra also contain a magnesium line and several other emission lines, such as $[\mathrm{O} \mathrm{III}] \lambda 5007$, but the $\mathrm{H} \alpha$ line is out of range. All this makes our comparative analysis of the quasar spectral properties limited mostly to the magnesium line. The value of the FWHM of $\mathrm{Mg}$ II line measured in all spectra (Table 1) indicates that it is a strong broad emission line typical for unobscured AGNs. However, the flux of the $\mathrm{Mg}$ II line shows great variability over these $19 \mathrm{yr}$, which indicates that it has responded to the continuum changes, although the scale of these responses differs between 

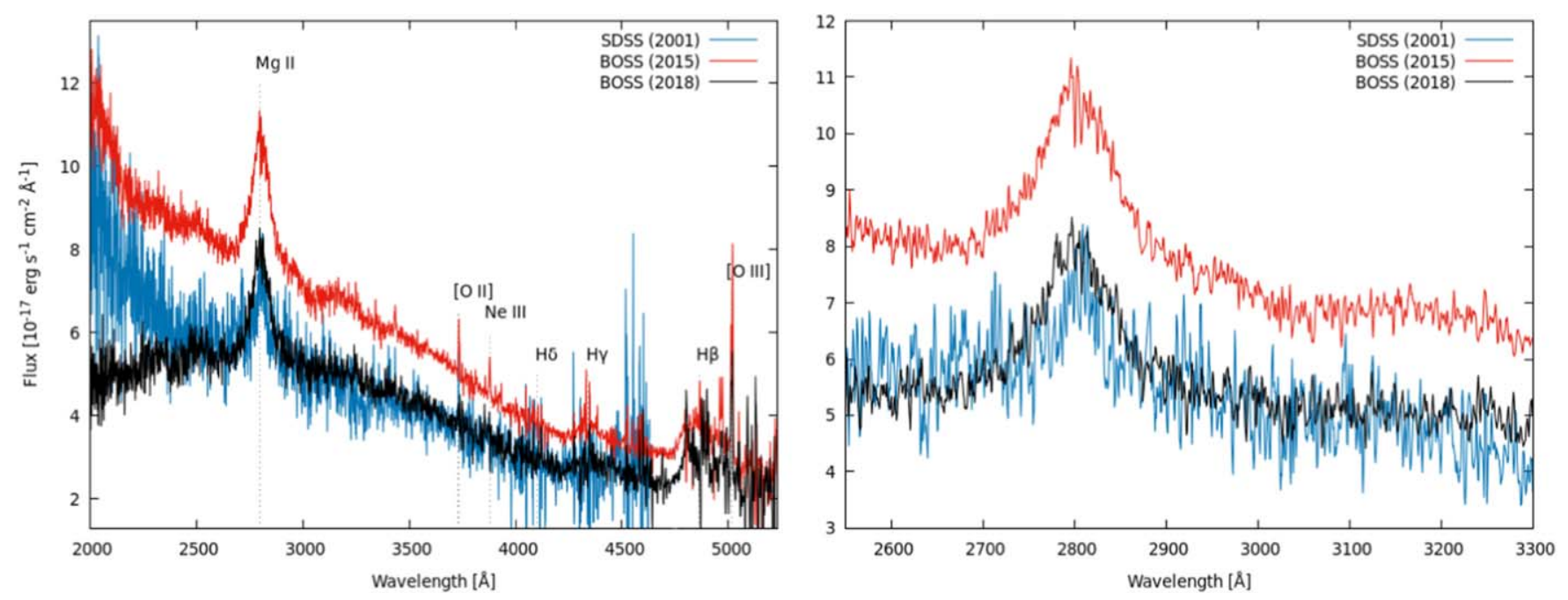

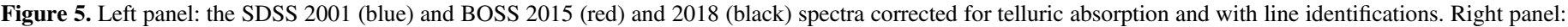
the $\mathrm{Mg}$ II line profile changes. The spectra were smoothed with a 3 pixel boxcar.

epochs. Both the continuum and the $\mathrm{Mg}$ II line flux increased significantly between 2001 and 2015 (Table 1). Over the next two years, the continuum decreased and in 2018 it reached the level observed in 2001 while the $\mathrm{Mg}$ II line did not change that much. The flux of the $\mathrm{Mg}$ II line showed only a slight decrease but still remained at a higher level compared to 2001. This probably implies a time lag with which the $\mathrm{Mg}$ II line reacts to continuum changes. We could not notice this for the continuum growth phase due to the lack of spectroscopic observations between 2001 and 2015. The time delay between the continuum variations and the response of the $\mathrm{Mg}$ II emission line has been already reported for radio-quiet (Czerny et al. 2019) and radio-loud objects (Nalewajko et al. 2019). What is more the very recent results on the study of a large sample of extremely variable radio-quiet AGNs (Homan et al. 2020) indicate that indeed the $\mathrm{Mg}$ II line does respond to the continuum changes, although the scale of behavior variability is very broad. In most of the studied AGNs the Mg II line varies less than the continuum. In the case of the quasar $013815+00$ the increase of the $\mathrm{Mg}$ II line flux observed in 2015 is greater than the change in the continuum emission, which indicates a more prominent response to the continuum changes than in typical AGNs. According to Guo et al. (2020) the Mg II line flux changes can be well explained as a photoionization response to extreme continuum variability. However, a wide range of possible responses among AGNs may indicate additional factors that affect the change in the Mg II line (Homan et al. 2020; Popović et al. 2019; Yang et al. 2020).

In the case of radio-loud AGNs, the complex behavior of the $\mathrm{Mg}$ II line described above further complicates the presence of a radio jet. There are detailed studies showing correlation between the jet kinematics and continuum and emission line variability in quasars and blazars suggesting that the jet affects the gas producing the broad emission lines and continuum (León-Tavares et al. 2013; Berton et al. 2018; Chavushyan et al. 2020). The scale of the nonthermal contribution may depend on the jet viewing angle and on the jet power. In order to estimate the contribution of the jet emission to the total optical-UV emission in the case of the quasar $013815+00$ we used the method proposed by Shaw et al. (2012). We calculated the value of the nonthermal dominance $\mathrm{NTD}=L_{\mathrm{obs}} / L p$ parameter, where $L_{\mathrm{obs}}$ is the observed continuum luminosity and $L_{p}$ is the predicted continuum luminosity estimated based on the continuum-line luminosity correlation found by
Shen et al. (2011) for a nonblazar sample. NTD parameter values calculated for both spectra (2015 and 2018) of the radio-loud phase of our quasar are very similar and do not exceed 1 $(\mathrm{NTD}<1)$. This may suggest that the observed continuum and $\mathrm{Mg}$ II emission in the radio-loud phase is reflecting the contribution from the accretion disk only.

\subsection{2. [O III] $\lambda 5007$ Line Properties}

Similarly, the radio jets of compact radio sources are thought to be strongly interacting with the denser phases of the interstellar medium in the near-nuclear regions of their host galaxies (Holt et al. 2008). Such strong jet-cloud interactions can cause the enhancement of the [O III] $\lambda 5007$ line emission as well as the radio emission boosting (Labiano 2008; Morganti et al. 2011). Additionally, the emission line kinematics of the CSS/GPS objects are more extreme in terms of line shifts and widths than the general populations of nearby AGN and radio galaxies with extended radio sources (Holt et al. 2008; Liao \& $\mathrm{Gu} 2020)$. In the case of quasar $013815+00$ only the spectroscopic BOSS $(2015,2018)$ observations allow us to characterize its $[\mathrm{O} \mathrm{III}] \lambda 5007$ line emission and follow its changes after the burst of radio activity. Unfortunately the low $\mathrm{S} / \mathrm{N}<5$ for $[\mathrm{O} \mathrm{III}] \lambda \lambda 4959,5007$ doublet prevented us from carrying out a more complex modeling of this emission.

The values of $[\mathrm{O}$ III $] \lambda 5007$ line luminosity (Table 1) and $5 \mathrm{GHz}$ radio luminosity from both epochs make the $013815+00$ follow the correlation between emission line luminosity and radio power of radio-loud AGNs (Kunert-Bajraszewska \& Labiano 2010; Morganti et al. 2011). This probably does not indicate any extra radio emission rising from the jet-cloud interactions but places the source among the so-called highexcitation galaxies on the plot. The value of $L_{[\mathrm{O} \text { III] }}$ is also the highest one when comparing it to the [O III] emission line luminosities of radio-quiet "changing-look AGNs" reported by MacLeod et al. (2016), which suggests that radio-loud 013815 +00 produces more ionizing photons. The FWHM of [O III] $\lambda 5007$ of $013815+00$ is also larger than the FWHM distribution peak for extended radio sources $\left(\sim 200-300 \mathrm{~km} \mathrm{~s}^{-1}\right.$,) and is in the range of values reported by Holt et al. (2008) for CSS/GPS sources. This may imply some impact of the jet on the narrow emission line region kinematics $(<1 \mathrm{kpc})$ in quasar $013815+00$. 
However, according to the high-resolution radio observations the radio jet of quasar $013815+00$ is small and the whole radio structure extends to tens of parsecs, which significantly limits the area of its impact unless the gas producing [O III] emission is located closer to the central black hole. Such an explanation has been discussed in the literature and the recent Hubble Space Telescope study of nearby Type 2 quasars shows that quasars with a more compact [O III] morphology have broader nuclear emission lines probably as a result of a gas concentration near the AGN (Fischer et al. 2018). This gas may be driven out at the later states by radio jets or AGN-winds, which may also operate on larger scales (100-1000s of pc). Without more detailed observations of the emission region in quasar $013815+00$ we are unable to distinguish any of the interpretations cited.

\subsection{Characteristic of the Accretion Process}

We used the measurements of the Mg II emission line and luminosity at $3000 \AA$ to estimate the black hole mass of quasar 013815+00 (Section 2.2 and Table 1). The obtained values for all epochs are consistent within the measurement errors. For further estimations we use the average black hole mass from the three epochs. In the next step we calculated the bolometric AGN luminosity (Table 1) in its radio-quiet stage using $\lambda L_{3000}$ and a conversion factor of 5.3 to convert from monochromatic to bolometric luminosity (Runnoe et al. 2012). With the Eddington luminosity defined as $L_{\mathrm{Edd}}=1.3 \times 10^{38} M_{\mathrm{BH}}$ and averaged black hole mass, we find the Eddington ratio $\left(\lambda_{\mathrm{Edd}}=L_{\mathrm{bol}} / L_{\mathrm{Edd}}\right)$ of 0.015 . If we estimate $L_{\mathrm{bol}}$ using the value of $\lambda L_{3000}$ from the BOSS (2015) spectrum after the source went into the radio-loud phase and again calculate the $\lambda_{\mathrm{Edd}}$, its value increases to 0.021 . However, during the next two years the luminosity and hence the Eddington ratio drops and again amounts to 0.015 , which is the same as in the radio-quiet phase.

The general formula defining bolometric luminosity says that $L_{\mathrm{bol}}$ is directly proportional to mass accretion rate $\dot{M}$ (in units of $M_{\odot} \mathrm{yr}^{-1}$ ) and the radiative efficiency $\eta$ of the accretion process: $L_{\mathrm{bol}}=\eta \dot{M} c^{2}$ (Coziol et al. 2017). Since the value of the $\mathrm{BH}$ mass of $013815+00$ probably does not change after the radio activity ignition we conclude that the observed increase in the Eddington ratio $\left(\sim \eta \dot{M} c^{2} / M_{\mathrm{BH}}\right)$ is a result of higher accretion rate and/or radiative efficiency. The change in the bolometric luminosity is not big (by a factor of 1.4) compared to the typical factor of 4 obtained for changing-look radio-quiet AGNs by MacLeod et al. (2019). Still, the relatively small change of the accretion rate and/ or radiative efficiency that took place in quasar $013815+00$, led to the launching of a new radio jet and a significant change in radio luminosity from $\log _{10}\left[L_{1.4 \mathrm{G} \mathrm{Hz}} / \mathrm{W} \mathrm{Hz}^{-1}\right]=24.1$ to 24.9 . Interestingly, the recent studies of the shape of the radio luminosity function (Malefahlo et al. 2020) show a change of its behavior in the luminosity range $\log _{10}\left[L_{1.4 \mathrm{G} \mathrm{Hz}} / \mathrm{W} \mathrm{Hz}^{-1}\right]=24-25$ what is interpreted as a change of the dominating population from radioquiet to radio-loud.

The estimated values of the black hole mass and Eddington ratio of $013815+00$ are in agreement with those calculated for other young AGNs, although the range of these values for AGNs considered young is very wide (Berton et al. 2017; Liao \& $\mathrm{Gu} 2020)$. The accretion power of $013815+00$ is larger than its jet power, which implies low jet efficiency $P_{j} / L_{\mathrm{bol}} \sim 0.02$ and makes the source comparable to flat-spectrum radio quasars and highexcitation FRII objects (Rusinek et al. 2017; Fan \& Wu 2019; Wójtowicz et al. 2020). The large black hole mass of $013815+00$ may suggest that the AGN is old and it probably has already gone through many such phases of enhanced accretion. We have only captured one of these phases. Perhaps this behavior could also be compared to the high/hard-to-soft state transition observed in black hole binaries and very recently also for a sample of young AGNs (Wójtowicz et al. 2020), with the relativistic jet being launched in a high state.

\section{Summary}

The burst of radio activity in quasar $013815+00$ detected on 2013 December 20 changed the source status from radio-quiet to radio-loud. The expanding new radio jet is responsible for the convex radio spectrum peaking at $\sim 5 \mathrm{GHz}$ in the early stage of activity but also for the rapid changes observed in this spectrum during the next few years. Ignition of radio emission coincides with a significant increase in the brightness of the accretion disk and emission lines. However, within the next two years, the disk brightness returns to its original state, which shows how fast changes occur in this new source. We suggest that the accretion disk behavior and birth of the radio source are the result of an enhancement of the accretion rate and/or radiative efficiency. The large black hole mass of $013815+00$ may indicate that such periods of increased accretion may have already occurred in the past for this object.

At gigahertz frequencies, the new radio source can currently be classified as a young flat-spectrum quasar. It went through a short GPS phase comparing to more powerful objects and its radio flux density has now stabilized at the level of a few millijansky. We predict that with such short-term jet activity, the development of the radio structure in this quasar will be very slow and therefore the source may remain compact for most of its life.

We thank the anonymous referee for helpful suggestions that led to improvement of the paper. We are grateful to Agnieszka Kuźmicz and Bożena Czerny for the useful discussion. The National Radio Astronomy Observatory is a facility of the National Science Foundation operated under cooperative agreement by Associated Universities, Inc. We thank the staff of the VLBA and VLA for carrying out these observations in their usual efficient manner. This work made use of the Swinburne University of Technology software correlator, developed as part of the Australian Major National Research Facilities Programme and operated under licence. M.K.B. and A.W. acknowledge support from the National Science Centre, Poland under grant no. 2017/26/E/ST9/00216. K.P.M. is a Jansky Fellow of the National Radio Astronomy Observatory.

Software: CASA (McMullin et al. 2007, AIPS (van Moorsel et al. 1996, IRAF (Tody 1986, 1993).

\section{ORCID iDs}

Magdalena Kunert-Bajraszewska (1) https://orcid.org/00000002-6741-9856

Kunal Mooley @i https://orcid.org/0000-0002-2557-5180

Preeti Kharb (i) https://orcid.org/0000-0003-3203-1613

\section{References}

An, T., \& Baan, W. A. 2012, ApJ, 760, 77

Baldi, R. D., Capetti, A., \& Giovannini, G. 2015, A\&A, 576, A38

Berton, M., Foschini, L., Caccianiga, A., et al. 2017, FrASS, 4, 8

Berton, M., Liao, N. H., La Mura, G., et al. 2018, A\&A, 614, 148

Bruhweiler, F., \& Verner, E. 2008, ApJ, 675, 83

Callingham, J. R., Ekers, R. D., Gaensleret, B. M., et al. 2017, ApJ, 836, 174 
Capetti, A., Baldi, R. D., Brienza, M., Morganti, R., \& Giovannini, G. 2019, A\&A, 631, 176

Chavushyan, V., Patiño-Álvarez, V. M., Amaya-Almazán, R. A., \& Carrasco, L. 2020, ApJ, 891, 68

Coziol, R., Andernach, H., Torres-Papaqui, J. P., Ortega-Minakata, R. A., \& Moreno del Rio, F. 2017, MNRAS, 466, 921

Czerny, B., Olejak, A., Rałowski, M., et al. 2019, ApJ, 880, 46

Czerny, B., Siemiginowska, A., Janiuk, A., Nikiel-Wroczyński, B., \& Stawarz, Ł. 2009, ApJ, 698, 840

Dallacasa, D., Stanghellini, C., Centonza, M., \& Fanti, R. , 2000, A\&A, 363,887

Drake, A. J., Djorgovski, S. G., \& Mahabal, A. 2009, ApJ, 696, 870

Fan, X., \& Wu, Q. 2019, ApJ, 879, 107

Fanaroff, B. L., \& Riley, J. M. 1974, MNRAS, 167, 31

Fanti, C., Fanti, R., Dallacasa, D., et al. 1995, A\&A, 302, 317

Fischer, T. C., Kraemer, S. B., Schmitt, H. R., et al. 2018, ApJ, 856, 102

Foschini, L. 2017, FrASS, 4, 6

Guo, H., Shen, Y., He, Z., et al. 2020, ApJ, 888, 58

Hardcastle, M. J., Williams, W. L., \& Best, P. N. 2019, A\&A, 622, 12

Hodge, J. A., Becker, R. H., White, R. L., Richards, G. T., \& Zeimann, G. R. 2011, ApJ, 142, 3

Holt, J., Tadhunter, C. N., \& Morganti, R. 2008, MNRAS, 387, 639

Homan, D., Macleod, C. L., Lawrence, A., Ross, N. P., \& Bruce, A. 2020, MNRAS, 496, 309

Keim, M. A., Callingham, J. R., \& Röttgering, H. J. A. 2019, A\&A, 628, 56

Kellerman, K. I., Sramek, R., Schmidt, M., Shaffer, D. B., \& Green, R. 1989, AJ, 98, 1195

Kharb, P., O'Dea, C. P., Baum, S. A., et al. 2014, MNRAS, 440, 2976

Kimball, A. E., Ivezić, Ž., Wiita, P. J., \& Schneider, D. P. 2011, AJ, 141, 182

Kunert-Bajraszewska, M., Gawroński, M. P., Labiano, A., \& Siemiginowska, A. 2010, MNRAS, 408, 2261

Kunert-Bajraszewska, M., \& Labiano, A. 2010, MNRAS, 408, 2279

Labiano, A. 2008, A\&A, 488, L59

Lähteenmäki, A., Järvelä, E., Ramakrishnan, V., et al. 2018, A\&A, 614, L1

LaMassa, S. M., Urry, C. M., Cappelluti, N., et al. 2013, MNRAS, 436, 3581L

León-Tavares, J., Chavushyan, V., Patiño-Álvarez, V., et al. 2013, ApJL, 763, L36

Liao, M., \& Gu, M. , 2020, MNRAS, 491, 92

MacLeod, C. L., Green, P. J., Anderson, S. F., et al. 2019, ApJ, 874, 8

MacLeod, C. L., Ross, N. P., Lawrence, A., et al. 2016, MNRAS, 457, 389

Malefahlo, E., Santos, M. G., Jarvis, M. J., White, S. V., \& Zwart, J. T. L., 2020, MNRAS, 492, 5297
McMullin, J. P., Waters, B., Schiebel, D., Young, W., \& Golap, K. 2007, in ASP Conf. Ser. 376, Astronomical Data Analysis Software and Systems XVI, ed. R. A. Shaw, F. Hill, \& D. J. Bell (San Francisco, CA: ASP), 127 Mooley, K. P., Hallinan, G., Bourke, S., et al. 2016, ApJ, 818, 105

Morganti, R., Holt, J., Tadhunter, C., et al. 2011, A\&A, 535, 97

Nalewajko, K., Gupta, A. C., Liao, M., et al. 2019, A\&A, 631, 4

O’Dea, C. P., \& Baum, S. A. 1997, AJ, 113, 148

Oke, J. B., \& Gunn, J. E. 1983, ApJ, 266, 713

Orienti, M., Dallacasa, D., Tinti, S., \& Stanghellini, C. 2006, A\&A, 450, 959

Padovani, P. , 2017, NatAs, 1, 0194

Popović, L. Č, Kovačević-Dojčinović, J., \& Marčeta-Mandić, S. 2019, MNRAS, 484, 3180

Readhead, A. C. S., Taylor, G. B., Xu, W., et al. 1996, ApJ, 460, 612

Reynolds, C. S., \& Begelman, M. C. 1997, ApJL, 487, L135

Runnoe, J. C., Brotherton, M. S., \& Shang, Z. 2012, MNRAS, 422, 478

Rusinek, K., Sikora, M., Kozieł-Wierzbowska, D., \& Godfrey, L. 2017, MNRAS, 466, 2294

Sadler, E. M., Ekers, R. D., Mahony, E. K., Mauch, T., \& Murphy, T. 2014, MNRAS, 438, 796

Schlafly, E. F., \& Finkbeiner, D. P. 2011, ApJ, 737, 103

Shaw, M. S., Romani, R. W., Cotter, G., et al. 2012, ApJ, 748, 49

Shen, Y., Richards, G. T., Strauss, M. A., et al. 2011, ApJS, 194, 45

Snellen, I. A. G., Schilizzi, R. T., Bremer, M. N., et al. 1999, MNRAS, 307, 149

Snellen, I. A. G., Schilizzi, R. T., de Bruyn, A. G., et al. 1998, A\&AS, 131, 435

Sotnikova, Yu., Mufakharov, T., Majorova, E., et al. 2019, AstBu, 74, 348

Tody, D. , 1986, Proc. SPIE, 627, 733

Tody, D. , 1993, in ASP Conf. Ser. 52, Astronomical Data Analysis Software and Systems II, ed. R. J. Hanisch, R. J. V. Brissenden, \& J. Barnes (San Francisco, CA: ASP), 173

Torniainen, I., Tornikoski, M., Teräsranta, H., Aller, M. F., \& Aller, H. D. 2005, A\&A, 435, 839

Trakhtenbrot, B., \& Netzer, H. 2012, MNRAS, 427, 3081

van Moorsel, G., Kemball, A., \& Greisen, E. 1996, in ASP Conf. Ser. 101, Astronomical Data Analysis Software and Systems V, ed. G. H. Jacoby \& J. Barnes (San Francisco, CA: ASP), 37

White, R. L., Becker, R. H., Helfand, D. J., \& Gregg, M. D. 1997, ApJ, 475,479

Wójtowicz, A., Stawarz, Ł., Cheung, C. C., et al. 2020, ApJ, 892, 116

Wołowska, A., Kunert-Bajraszewska, M., Mooley, K., \& Hallinan, G. 2017, FrASS, 4, 38

Yang, Q., Shen, Y., Chen, Y., et al. 2020, MNRAS, 493, 5773 